\title{
Erratum to: An optimal inventory management problem with reputation-dependent demand
}

\author{
Eugene Khmelnitsky $^{1}$. Gonen Singer ${ }^{2}$
}

Published online: 26 March 2015

C Springer Science+Business Media New York 2015

\section{Erratum to: Ann Oper Res DOI 10.1007/s10479-014-1600-z}

We would like to make the following clarifications and corrections to the original paper.

1. In this paper, we derived necessary optimality conditions for the stated problem. Therefore, the phrase "optimal solution" used in the paper should have the meaning of local optimum.

2. The argument proven in Lemma 2 is not sufficient for global optimality of a reputationdependent base-stock policy. Generally, the globally optimal policy may depend on the two state variables: $x$-the inventory level and $r$-the value of reputation, rather than only on $r$ as shown in the paper. Consequently, the statement that the optimal solution is a reputation-dependent base-stock policy is incorrect.

In case the optimal policy depends on the two state variables, the necessary optimality conditions (18) and (19) remain without change, except that $y(r)$ and $\psi_{2}(r)$ are replaced by $y(r, x)$ and $\psi_{2}(r, x)$, as follows:

$$
\begin{aligned}
& (p-c)\left(1-\beta E_{\pi}\left[F^{\prime}(y(r, x)-r \xi)\right]\right)=E_{\pi}\left[C^{\prime}(F(y(r, x)-r \xi)) F^{\prime}(y(r, x)-r \xi)\right] \\
& \quad+\beta(1-\gamma) E_{\pi}\left[G^{\prime}(F(y(r, x)-r \xi)) F^{\prime}(y(r, x)-r \xi) \psi_{2}(\gamma r\right. \\
& \quad+(1-\gamma) G(F(y(r)-r \xi)), F(y(r, x)-r \xi))]
\end{aligned}
$$

The online version of the original article can be found under doi:10.1007/s10479-014-1600-z.

Eugene Khmelnitsky

xmel@tau.ac.il

Gonen Singer

singerg@afeka.ac.il

1 Department of Industrial Engineering, Tel-Aviv University, 69978 Ramat-Aviv, Israel

2 Department of Industrial Engineering and Management, Afeka College, 69107 Tel-Aviv, Israel 


$$
\begin{aligned}
\psi_{2}(r, x)= & -E_{\pi}\left[\xi F^{\prime}(y(r, x)-r \xi)\left(C^{\prime}(F(y(r, x)-r \xi))+\beta(p-c)\right)\right] \\
& +\beta E_{\pi}\left[\left(\gamma-(1-\gamma) \xi G^{\prime}(F(y(r, x)-r \xi)) F^{\prime}(y(r, x)-r \xi)\right) \psi_{2}(\gamma r\right. \\
& +(1-\gamma) G(F(y(r)-r \xi)), F(y(r)-r \xi))] \text { for } x \leq y(r, x)
\end{aligned}
$$

3. In case the optimal solution exists and the necessary optimality conditions, whether in the form of (18) and (19), or in the form of (18a) and (19a), have a unique solution, it is globally optimal.

4. Since Sect. 4.1 presents three limit cases, in the phrase "Below, we present analytical solutions for two limit cases of (18) and (19) followed by numerical examples," "two" should be replaced by "three."

5. The correct form of Eq. (20) is

$$
(p-c)\left(1-\beta E_{\pi}\left[F^{\prime}(y(r)-r \xi)\right]\right)=E_{\pi}\left[C^{\prime}(F(y(r)-r \xi)) F^{\prime}(y(r)-r \xi)\right] .
$$

This equation is true for both full backlogging $(\alpha=1)$ and no backlogging $(\alpha=0)$ cases, rather than for full backlogging only, as printed.

6. In Eq. (23), we assumed, without loss of generality, that $\xi_{1}<\xi_{2}$.

We thank Prof. Ye Lu for pointing out that the argument of Lemma 2 does not prove the optimality of a reputation-dependent base-stock policy, and that the optimal policy may depend on both $x$ and $r$. 